\title{
Quantitative Assessment Method Of Rock Burst
}

\author{
LIU Bin ${ }^{1, \text { a }}$, Peng Xing-xin ${ }^{1, b}$, LIU Quan-sheng, ${ }^{1, c}$, Huang \\ Kai-zhong, ${ }^{2, \mathrm{~d}}$ \\ ${ }^{1}$ State Key Laboratory of Geomechanics and Geotechnical \\ Engineering, Institute of Rock and Soil Mechanics, Chinese Academy of \\ Sciences, Wuhan,430071, China \\ ${ }^{2}$ Huainan Mine industry Company, State Key Laboratory of Deep Coal \\ Mining and Environmental Protection, Huainan 232001, China \\ abinliu81@126.com, ${ }^{b}$ pengxx1990@163.com, ${ }^{c}$ liuqs@whrsm.ac.cn, ${ }^{d}$ Scb \\ 2003@126.com
}

\begin{abstract}
Rock burst is one of the three big power disasters in deep mining environment and its quantities assessment method study is a hotspot issue. The analytical mode and method is set up for analysis of rock burst quantitative assessment in Deep Mining environment stress in this paper. The influence and sensibility of relative factors such as stress level, the size and damage degree of EDZ, Poisson's ratio and stress drop factors on the rock burst tendency and released energy during rock burst are discussed systematically. The research result indicate that: 1) with constant size of damage zone, the more rock damage, the more difficultly rock burst occur; 2) the rock burst tendency is insensitive to Poisson's ratio change; 3) with constant higher value of damage degree, the smaller damage zone size, the more difficultly rock burst occur, and there is inverse results in constant smaller value of damage degree condition.
\end{abstract}

Keywords: rock burst tendency, analytical mode, sensibility, energy release

\section{Introduction}

Rock burst is one of the three big power disasters in deep mining environment accompanied by a violent shock mainly felt and can cause significant damage. Mining operations induce a redistribution of the stress environment based on the mechanical behavior of surrounding rock. In terms of surrounding rock with rock burst tendency, the rock burst occur when elastic energy accumulation of rock mass system for rock mass deformation reached the limit of rock mass energy storage[1], and the condition that the factors effected elastic energy accumulation needed to meet can be laid out. The rock burst tendency analysis can be defined as a progress as following: 1) making sure the stress condition of surrounding rock and the characteristic of relevant factors which effect elastic energy accumulation; 
2) making hazard assessment of rock burst through comparing the factors that affect system elastic energy accumulation in particular situation with that of rock burst occurrence. The form of released energy during rock burst evolution progress show as two type mainly[2]: dissipate energy caused by crack initiation and growth before peak strength and the kinetic energy for brittle stress drop after peak strength. The dissipate energy as a certain parameter has been closely related to system elastic energy accumulation, and rock burst brisance is determined by the kinetic energy for brittle stress drop.

For the purpose of forecasting rock burst effectively, the problems of rock burst tendency need to be analyzed theoretically, analytical model should be put forward for the better understanding about rock burst occurrence mechanical mechanism of surrounding rock system, and the effective rock burst prediction index can be put forward. In this paper, taking the Granite circular tunnel for example, the influence laws of rock burst tendency affected by relative parameters in equipressure condition with the analytical method are researched theoretically Organization of the Text

\section{Analytical model for rock burst tendency}

From the discussion about rock burst constitutive form, the following analysis of tunnel surrounding rock is ease. For the surrounding rock of Granit tunnel, the minimum principal stress of surrounding rock stress is zero at excavation face, and increase parting from surrounding rock, reach to initial rock stress state finally. The rock burst constitutive type is changed with confining stress[3]: Under lower confining stress condition, the excavation damage zone (EDZ) of surrounding rock shown as Fig.1 (a) is annular region between radius a and radius c (mentioned as annular ac in following paper), and the constitutive form for this condition is elastic-damage-elastic-brittle-idea plastic type. The damage and the energy accumulation, transfer and release in annulus ac surrounding rock are induced by energy input from outside surrounding rock. Under higher confining stress condition the constitutive form of surrounding rock is elastic-damage-elastic-brittle-idea plastic form in the annulus ac near excavation surface and elastic-brittle-idea plastic form in the annulus cb far away excavation surface as shown in Fig.1(b). The input energy of annulus ac from outside surrounding rock come from elastic energy accumulated in excavated rock and energy result from elastic deformation of annulus cb.

So the EDZ of surrounding rock is annulus ac zone, which is the key zone for rock burst tendency analysis[4]. For the purpose of theoretical analysis of rock burst tendency in the key zone, the analytical model is set up as shown in Fig.2. The inside radius and outside radius of the key zone (annulus ac) is a and $r$ respectively. $\sigma_{r c}$ and $\tau_{r \theta}$ are the normal stress and shear stress that outside surrounding rock act on the key zone respectively. The internal forces of annulus ac are circumference force $\mathrm{T}$ and moment $\mathrm{M}$. 


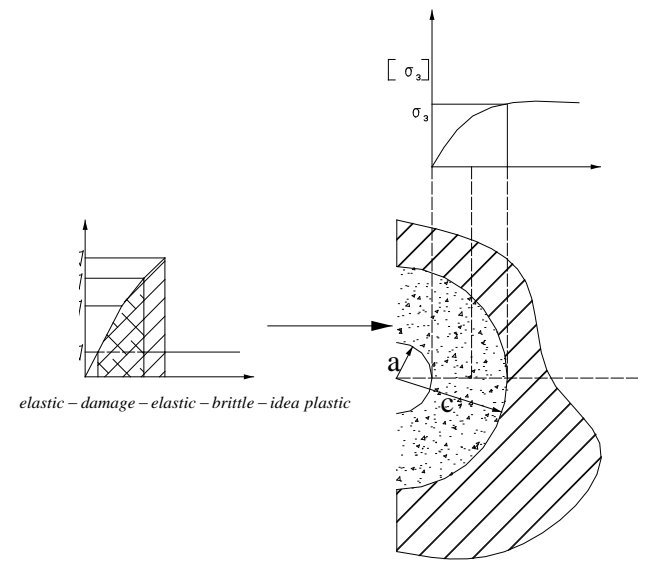

(a) lower confining stress condition

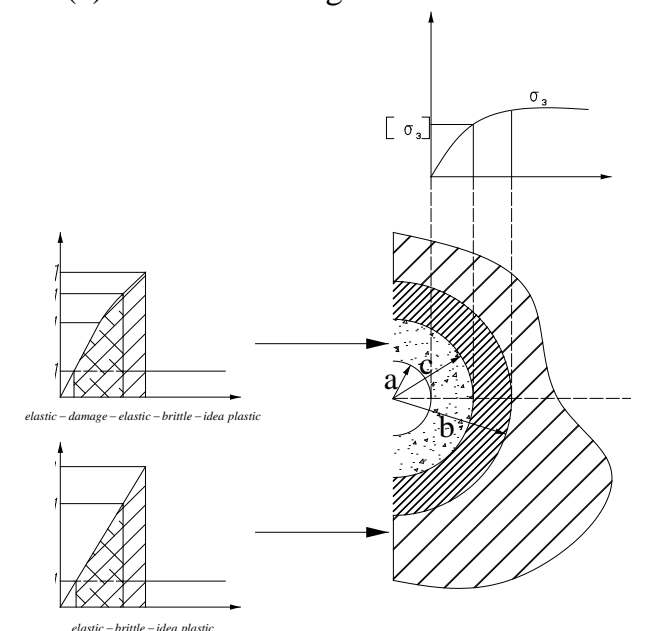

(b) higher confining stress condition

Fig.1 The excavation damage zone (EDZ) of surrounding rock

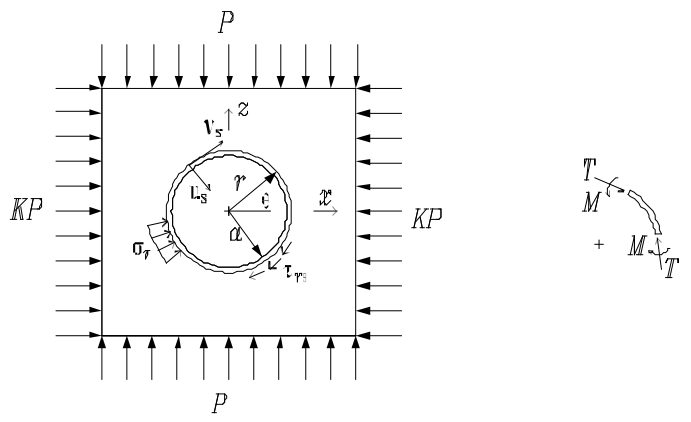


Fig.2 analytical model of rock burst tendency

For the rock with rock burst tendency, the major fracture type is brittle tensile fracture during the rock burst evolution progress, and plastic dissipative energy produced by shear failure is less. So the hypothesis is put forward that there are two type modes during rock burst evolution: no shear stress mode and no shear displacement mode. The analytical solutions for the modes mentioned above are derived in this paper.

(1) Analytical solutions for no shear displacement mode

$$
\begin{gathered}
u_{r}=\frac{P(1+\mu) r}{E_{r}}\left\{\frac{1}{2}(1+K) a_{0}+\frac{1}{2}(1-K)\left[4(1-\mu) b_{2}-2 a_{2}\right] \cos 2 \theta\right\} \\
u_{\theta}=\frac{P(1+\mu) r}{E_{r}}\left\{-(1-K)\left[a_{2}+(1-2 \mu) b_{2}\right] \sin 2 \theta\right\} \\
T=\frac{P}{2} r\left\{(1+K)\left(1-a_{0}\right)+(1-K)\left[1+2 a_{2}\right] \cos 2 \theta\right\} \\
M=\frac{P}{2} r^{2}\left\{\frac{1}{2}(1-K)\left[1-2 a_{2}+2 b_{2}\right] \cos 2 \theta\right\} \\
\sigma_{r}=\frac{P}{2}\left\{(1+K)\left(1-a_{0}\right)-(1-K)\left[1-6 a_{2}+4 b_{2}\right] \cos 2 \theta\right\} \\
\tau_{r \theta}=\frac{P}{2}\left\{(1-K)\left[1+6 a_{2}-2 b_{2}\right] \sin 2 \theta\right\}
\end{gathered}
$$

Where, $\theta$ is the angle between calculation point direction and horizontal direction; $\mathrm{P}$ is the vertical stress; $\mathrm{T}$ is the axial force in annulus ac, $\mathrm{M}$ is the bending moment of micro-segment shown in Fig.2, $\mathrm{K}$ is the ratio horizontal stress to vertical stress; $E_{r}$ is the elastic modulus of outside annulus ac; $E_{a}$ is the elastic modulus of annulus ac; $\mu$ is the Poisson ratio; $\sigma_{r}$ is the normal stress, $a_{0}, a_{2}, b_{2}$ are no dimensional parameters, and its mathematical expressions are shown as follow:

$$
\begin{gathered}
a_{0}=\frac{C F(1-\mu)}{C+F+C F(1-\mu)} \\
a_{2}=\beta b_{2}, \quad b_{2}=\frac{C(1-\mu)}{2[C(1-\mu)+4 \mu-6 \beta-3 \beta C(1-\mu)]} \\
\quad \beta=\frac{(6+F) C(1-\mu)+2 F \mu}{3 F+3 C+2 C F(1-\mu)} \quad C=\frac{E_{r} r}{E_{a}(r-a)} \quad, \quad F=\frac{12 E_{r} r^{3}}{E_{a}(r-a)^{3}}
\end{gathered}
$$

(2) analytical solutions for no shear stress mode

$$
\begin{array}{r}
u_{r}=\frac{P(1+\mu) r}{E_{r}}\left\{\frac{1}{2}(1+K) a_{0}^{*}-(1-K)\left[(5-6 \mu) a_{2}^{*}-(1-\mu)\right] \cos 2 \theta\right\} \\
u_{\theta}=\frac{P(1+\mu) r}{E_{r}}\left\{\frac{1}{2}(1-K)\left[(5-6 \mu) a_{2}^{*}-(1-\mu)\right] \sin 2 \theta\right\}
\end{array}
$$




$$
\begin{gathered}
T=\frac{P}{2} r\left\{(1+K)\left(1-a_{0}^{*}\right)+(1-K)\left[1+2 a_{2}^{*}\right] \cos 2 \theta\right\} \\
M=\frac{P}{2} r^{2}\left\{(1-K)\left[1-2 a_{2}^{*}\right] \cos 2 \theta\right\} \\
\sigma_{r}=\frac{P}{2}\left\{(1+K)\left(1-a_{0}^{*}\right)-(1-K)\left[3-6 a_{2}^{*}\right] \cos 2 \theta\right\} \quad \tau_{r \theta}=0
\end{gathered}
$$

$a_{0}^{*}, a_{2}^{*}$ are no dimensional parameters, and its mathematical expressions are shown as follow:

$$
a_{0}^{*}=\frac{C F(1-\mu)}{C+F+C F(1-\mu)} \quad a_{2}^{*}=\frac{(F+6)(1-\mu)}{2 F(1-\mu)+6(5-6 \mu)}
$$

\section{The rock burst tendency with equipressure}

Under equipressure condition $K=1$, the analytical results of no shear stress mode and no shear displacement mode are equal and can be deduce from Eq.2. The analytical results is shown as Eq.3.

$$
u_{r}=\frac{P(1+\mu) r}{E_{r}} a_{0}^{*}, \quad u_{\theta}=0, T=\operatorname{Pr}\left(1-a_{0}^{*}\right), \quad M=0, \sigma_{r}=P\left(1-a_{0}^{*}\right), \quad \tau_{r \theta}=0
$$

Taking annular ac for research object, the energy storaged in annular is equal to the external work $W$ shown as Eq.4.

$$
W=2 \pi r \sigma_{r} \frac{u_{r}}{2}=\pi \frac{P^{2}(1+\mu) r^{2}}{E_{r}}\left(1-a_{0}^{*}\right) a_{0}^{*}
$$

At the excavation surface, the normal stress is zero, the stressing model of arbitrary micro-segment of annular ac belong to uniaxial compression state[5]. Making the central angle of arbitrary micro-segment as $d \theta$, then the expression of lengths $\mathrm{L}$ of micro-segment is $(a+r) d \theta / 2$ then expression of axial stress $\sigma_{\theta}$ act on the micro-segment, caused by axial force $\mathrm{T}$, is $T /(r-a)$ The expression of rock limit energy storage density under uniaxial compression without damage is defined as Eq.5.

$$
[\phi]=\sigma_{c}^{2} / 2 E_{r}
$$

Then the expression of limit storage energy $[U]$ of annular ac is Eq.6.

$$
[U]=\int_{0}^{2 \pi} \int_{a}^{c}[\phi] r d r d \theta=\frac{\pi \sigma_{c}^{2}}{2 E_{r}}\left(r^{2}-a^{2}\right)
$$

The rock burst occurrence condition is $W=[U]$, it can be expressed as Eq.7 deduced from Eq.5 and Eq.6. 


$$
\left(\frac{P}{\sigma_{c}}\right)^{2}=\frac{\left(1+\frac{a}{r}\right)\left[\frac{1}{12}\left(\frac{E_{a}}{E_{r}}\right)\left(1-\frac{a}{r}\right)^{3}+\frac{E_{a}}{E_{r}}\left(1-\frac{a}{r}\right)+(1-\mu)\right]^{2}}{\left(1-\mu^{2}\right)\left(\frac{1}{6}\left(\frac{E_{a}}{E_{r}}\right)\left(1-\frac{a}{r}\right)^{2}+2 \frac{E_{a}}{E_{r}}\right)}
$$

And analysis figure of rock burst tendency is obtained with the stress ratio $P / \sigma_{c}$ as the abscissa and the $a / r$ as the ordinate from Eq.7. The $P / \sigma_{c}$ reflect the stress level of rock burst occurrence, and $a / r$ reflect the size of damage zone. Variable $E_{a} / E_{r}$ in Fig.3 (a) reflect the damage degree of damage zone. The influence law of rock burst tendency affected by Poisson's ratio is shown in Fig.3 (b).

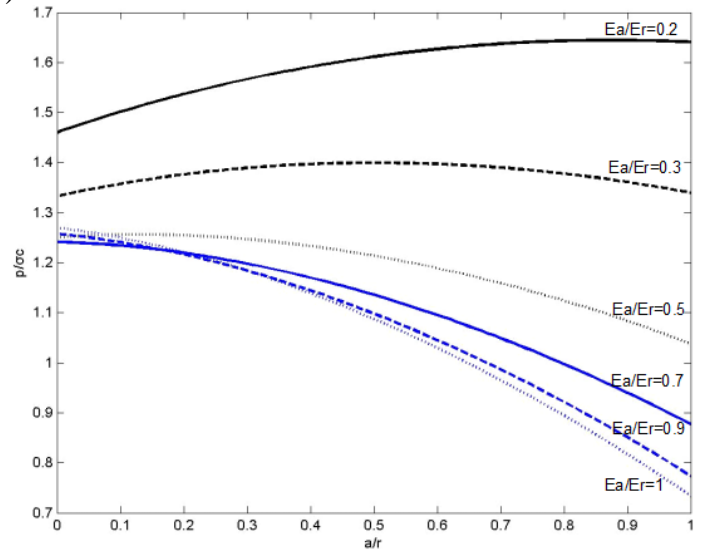

(a)

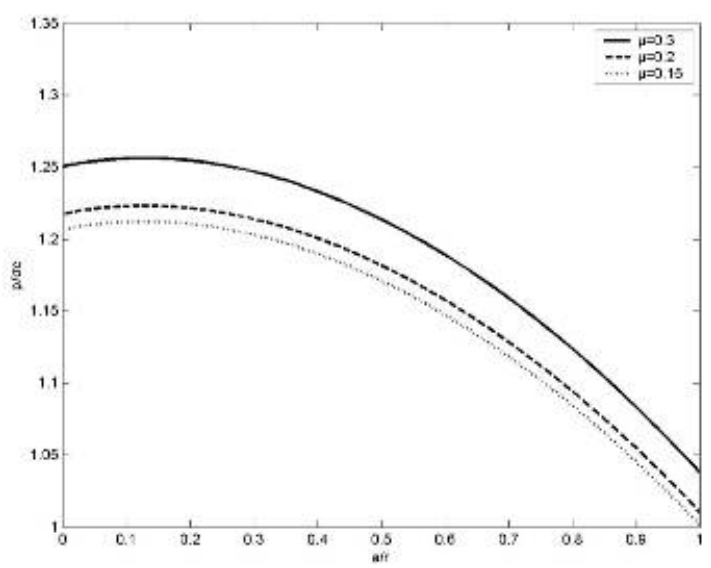

(b)

Fig.3 Rock burst tendency analysis figure 
The three influence laws of rock burst tendency affected by relative deformation parameters with equipressure reflected by Fig.3 is put forward as following:

1) With constant size of damage zone, the more rock damage in annulus ac (the smaller value of $E_{a} / E_{r}$ ), the more difficultly rock burst occur for the higher stress level (the bigger value of $P / \sigma_{c}$ )needed to induce rock burst;

2) the rock burst tendency is affected by Poisson's ratio but is insensitive to it, the smaller value of Poisson's ratio $\mu$, the more difficultly rock burst occur;

3) With constant bigger value of damage degree, the smaller damage zone size, the more difficultly rock burst occur. And there are inverse influence laws of rock burst tendency affected by damage zone size with constant smaller value of damage degree.

\section{Conclusions}

1) Analytical model of rock burst tendency is set up, the influence laws of rock burst tendency affected by relative deformation parameters and energy release laws during rock burst is obtained.

2) With constant size of damage zone, the more rock damage, the more difficultly rock burst occur the rock burst tendency is insensitive to Poisson's ratio change; with constant bigger value of damage degree, the smaller damage zone size, the more difficultly rock burst occur. And there are inverse influence laws of rock burst tendency affected by damage zone size with constant smaller value of damage degree.

\section{Acknowledgements}

This work is supported by the National Natural Science Foundation of China under the research with the grant No. 51474205, 41102198 and the Key Research Program of the Chinese Academy of Sciences, with the grant No. KZZD-EW-05.

\section{References}

[1] Hua An-zeng. Chinese Journal of Rock Mechanics and Engineering,Vol.22(2003),p.1054-1059 
[2] Hajiabdolmajid V, Kaiser P K, Martin C D. Int J Rock Mech Mining Sci.Vol.39(2002),p.739-741.

[3] Liu Bin, Liu Quan-sheng. Advanced Science Letters. Vol.4(2011),p. 2267-2271.

[4] R.A.Stewart, W.U.Reimold. TECTONOPHYSICS. Vol.337(2001),p.173-193

[5] Erik Eberhardt. Brittle rock fracture and progressive damage in uniaxial compression [D], 1999. 